\title{
Editorial \\ Crop Genetic Resources: An Overview
}

\author{
Gregorio Barba-Espin $1, *(\mathbb{D})$ and José Ramón Acosta-Motos $1,2, * \mathbb{C}$ \\ 1 Group of Fruit Biotechnology, Department of Fruit Breeding, CEBAS-CSIC, 30100 Murcia, Spain \\ 2 Campus de Los Jerónimos, Universidad Católica San Antonio de Murcia, 30107 Guadalupe, Spain \\ * Correspondence: gbespin@cebas.csic.es (G.B.-E.); jracosta@ucam.edu (J.R.A.-M.)
}

check for

updates

Citation: Barba-Espin, G.;

Acosta-Motos, J.R. Crop Genetic

Resources: An Overview. Agronomy 2022, 12, 340. https://doi.org/ 10.3390 /agronomy 12020340

Received: 20 December 2021

Accepted: 27 January 2022

Published: 29 January 2022

Publisher's Note: MDPI stays neutral with regard to jurisdictional claims in published maps and institutional affiliations.

Copyright: (C) 2022 by the authors. Licensee MDPI, Basel, Switzerland. This article is an open access article distributed under the terms and conditions of the Creative Commons Attribution (CC BY) license (https:// creativecommons.org/licenses/by/ $4.0 /)$.
The impacts of climate change on crop production are already a reality worldwide. Extreme weather events such as droughts, floods and heat waves are increasingly frequent, and this is affecting agriculture due to its high vulnerability. In order to mitigate these impacts, the access to unexploited genetic crop diversity for the production of new varieties which can thrive in more extreme environmental conditions is of prime importance. A multitude of beneficial agronomic traits have been lost over the course of domestication, and, in this sense, crops' wild relatives and landraces contain many genes of potential value for plant breeding. Among these, there are many traits that are relevant for climate change adaptation. Despite the vast pool of resources that exists, much of the crop germplasm richness found in gene banks is underutilized. To overcome the barriers between germplasm conservation and use, evaluation is necessary to discover the useful diversity they contain.

The number of accessions deposited in germplasm banks is continuously growing. Germplasm evaluation refers to the observation, measurement and reporting of heritable plant traits from a collection. The Food and Agriculture Organization of the United Nations stressed, in a recent report, that one of the major constrains affecting the conservation, use, monitoring and reporting of information on plant genetic resources for food and agriculture (PGRFA) concerns data access and communication among researchers, breeders, farmers and governments. In this sense, considering the broad range found in the germplasm collections of cultivated plants, which varies from wild and weedy types to high-yielding varieties, it is of major importance to ensure an accessible standardized format for data compilation and management. Other shared problems rely on developing sampling strategies for representative specimens in natural habitats or the design of technologies for long-term maintenance.

To date, germplasm evaluation has been centered mostly on morphological descriptors, agronomical traits and molecular marker technology. In spite of their reliability, low cost and accessibility, morphological descriptors present some constraints, such as the influence of environment on the genotype, making evaluation and information exchange more complex, and limited polymorphism, which demands the evaluation of more descriptors. On the other hand, evaluation based on agronomic characters is especially used in economically relevant crops, although a complete evaluation in this respect is costly, time-consuming and labor-intensive; as a consequence, nowadays it covers a small fraction of the accessions of interest. However, the final aim of germplasm evaluation and plant breeding is to obtain desirable traits in a highly efficient manner. Thus, traditional evaluation techniques are not sufficient to fulfil demands on food security and sustainability.

Crop performance is determined by complex traits resulting from genetics and epigenetics interactions. Understanding the relationship between genotype and phenotype is important for the sustainable evaluation and conservation of crop germplasm richness. Thus, traditional evaluation techniques are nowadays giving way to germplasm characterization based on molecular methods. In this sense, molecular markers, which reveal DNA sequence polymorphism, constituted a turning point in germplasm characterization. However, approaches based on molecular markers ultimately have the limitation 
of considering genes as independent functional entities; when traits are the result of a multigenic regulation and have a close interaction with environmental conditions, broader approaches are needed. Nowadays, the rapid expansion of the "-omics" techniques are bringing high-throughput approaches to the frontline of plant breeding, accelerating crop improvement by elucidating the interaction between genotype and phenotype. In this sense, epigenomics, genomics, transcriptomics, proteomics, metabolomics, phenomics and ionomics, together with bioinformatic tools, have made it possible for breeders to design more resilient and/or more productive varieties towards biotic and abiotic stresses. More recently, multi-omic approaches have been proposed for plant evaluation and breeding, integrating datasets from the different -omics together with complex mathematical models.

Through this Special Issue, there are examples of such complexity and diversity of germplasm evaluation on different cash crop species such as carrot, barley, soybean, almond, melon, lettuce, tomato, wheat, cacao and rice [1-11]. Acosta-Motos et al. [1] conducted agromorphological characterization on Eastern carrot landraces; these may be a source of material for carrot breeding programs, particularly black carrot accessions due to their high anthocyanin and flavonoid contents and, concomitantly, antioxidant capacity. In this sense, commercial cultivars are, overall, more productive than landraces; however, landraces are becoming significant sources of genetic variability and a crucial element for agrobiodiversity due to their high variation and adaptability to local environments. Dziurdziak et al. [2] conducted a genome-wide DArTseq analysis of the diversity of 116 spring barley landraces from different countries, preserved in the collection of the Polish gene bank; this revealed remarkable variation among landraces related to the country of origin and the grain type, and supporting the breeding of hulless accessions for spring barley's high quality as a food with beneficial health values.

The interaction between genotype and environment was studied by Abdelghany et al. [3], where the genotypic stability of 135 soybean accessions was evaluated in three Chinese locations. This was achieved by means of mathematical models with a weighted average of absolute scores biplot and a multi-trait stability index. The latter tool proved to be very useful in finding genotypes suitable for both seed performance and stability, by analyzing seven seed composition traits simultaneously. Soybean germplasm diversity was also explored by Jo et al. [4], who analyzed 470 soybean accessions of black seed coat and green cotyledons-rich in chlorophylls, anthocyanin and compounds with anticarcinogenic properties - by $6 \mathrm{~K}$ single nucleotide polymorphic loci, to determine genetic architecture. As a result, 36 accessions were found to contain $99.5 \%$ of the diversity from the total collection analyzed, showing potential for their use in breeding programs.

Twenty-four traditional almond cultivars clearly in decline or close to extinction were analyzed by Pérez- Sánchez et al. [5], from agromorphological and nutritional points of view, using a total of 40 descriptors gathered in international guidelines such as IPGRI and UPOV for flowers, leaves, fruits and vegetative tree habits. As a result, certain cultivars were found to have a high yield and quality of fruit, which constitutes an important step in the conservation of genetic almond resources in the Central-Western Iberian Peninsula.

The application of molecular markers to assess the genetic variability of plant varieties and cultivars is well represented in this Special Issue. Chikh-Rouhou et al. [6] combined 24 phenotypic traits and eight Simple Sequence Repeat (SSR) molecular markers to assess the genetic diversity of a Tunisian melon collection. A considerable phenotypic variability among accessions was measured for several traits of agronomical importance, whereas all of the microsatellites were found to be polymorphic. A precise clustering for landraces and breeding lines was obtained using combined phenotypic-molecular data, which may allow the correct use of these accessions in future breeding programs. Similarly, Caramante et al. [7] also utilized SSR markers to compare 15 traditional landraces with 15 widely used current varieties of tomato, concluding from the data analysis that the landraces conformed to a genetically different population from the commercial varieties, and serving as a milestone for implementing in situ and ex situ conservation programs. Another type of molecular marker, high-density single-nucleotide polymorphism (SNP), has been 
used by Ganugi et al. [8] to assess the genetic variability of a 265-accession collection of eight tetraploid wheat subspecies. The analysis and data treatment revealed clusters in agreement with the taxonomic classification, shedding light on the wheat's evolutionary history and the phylogenetic relationships among subspecies.

Around $90 \%$ of the cacao's production worldwide is located in developing countries, especially in small farming systems. Criollo cacao is highly demanded in Honduras due to is quality attributes. López et al. [9] used 16 SSR molecular markers on 89 samples showing phenotypic traits of Criollo cacao, to assay purity and belonging to that group. As a result, although certain accessions had the genetic traits of Trinitario or other admixtures of cacao types, the genetic purity of Criollo cacao in Honduras was confirmed, providing further evidence of Mesoamerica as a cacao domestication center.

Cultivated lettuce is one of the main leafy vegetables worldwide. Birlanga et al. [10] established, based on a hydroponic system, a root and shoot phenotyping of 12 lettuce genotypes. Tipburn incidence and leaf nutrient content were analyzed, identifying nutrient traits highly correlated with genotype-dependent tipburn, which may lead, in defined nutrient solutions, to select for tipburn-tolerant genotypes that could be adequate for hydroponic cultivation. Chan-in et al. [11] evaluated grain quality traits and allelic variation of the Badh2 gene-determining fragrance attributes-in 22 Thai rice landraces. Haplotype analysis on the Badh2 gene revealed a correlation with grain aroma by sensory evaluation. The results indicated that genetic resources could be introduced for fragrant rice breeding programmes to increase the income of highland farmers.

Taken together, the present Special Issue contributes to the efforts on crop germplasm evaluation in order to ensure future food security and commercial profitability. Nevertheless, plant genetic biodiversity is under threat from genetic erosion, a concept introduced by researchers to describe the gradual loss of individual genes and combinations of genes, such as those present in domesticated landraces. The major cause of genetic erosion is the displacement of landraces by contemporary varieties. To overcome these problems, ex situ and in situ conservation approaches must be undertaken. Likewise, the role of indigenous and farming peoples in such efforts should be considered for the preservation of crops' wild relatives and landraces.

Author Contributions: Writing — original draft preparation, G.B.-E. and J.R.A.-M.; writing-review and editing, G.B.-E. and J.R.A.-M. All authors have read and agreed to the published version of the manuscript.

Conflicts of Interest: The authors declare no conflict of interest.

\section{References}

1. Acosta-Motos, J.R.; Díaz-Vivancos, P.; Becerra-Gutiérrez, V.; Hernández Cortés, J.A.; Barba-Espín, G. Comparative Characterization of Eastern Carrot Accessions for Some Main Agricultural Traits. Agronomy 2021, 11, 2460. [CrossRef]

2. Dziurdziak, J.; Gryziak, G.; Groszyk, J.; Podyma, W.; Boczkowska, M. DArTseq Genotypic and Phenotypic Diversity of Barley Landraces Originating from Different Countries. Agronomy 2021, 11, 2330. [CrossRef]

3. Abdelghany, A.M.; Zhang, S.; Azam, M.; Shaibu, A.S.; Feng, Y.; Qi, J.; Li, J.; Li, Y.; Tian, Y.; Hong, H.; et al. Exploring the Phenotypic Stability of Soybean Seed Compositions Using Multi-Trait Stability Index Approach. Agronomy 2021, 11, 2200. [CrossRef]

4. Jo, H.; Lee, J.Y.; Cho, H.; Choi, H.J.; Son, C.K.; Bae, J.S.; Bilyeu, K.; Song, J.T.; Lee, J.-D. Genetic Diversity of Soybeans (Glycine max (L.) Merr.) with Black Seed Coats and Green Cotyledons in Korean Germplasm. Agronomy 2021, 11, 581. [CrossRef]

5. Pérez-Sánchez, R.; Morales-Corts, M.R. Agromorphological Characterization and Nutritional Value of Traditional Almond Cultivars Grown in the Central-Western Iberian Peninsula. Agronomy 2021, 11, 1238. [CrossRef]

6. Chikh-Rouhou, H.; Mezghani, N.; Mnasri, S.; Mezghani, N.; Garcés-Claver, A. Assessing the Genetic Diversity and Population Structure of a Tunisian Melon (Cucumis melo L.) Collection Using Phenotypic Traits and SSR Molecular Markers. Agronomy 2021, 11, 1121. [CrossRef]

7. Caramante, M.; Rouphael, Y.; Corrado, G. The Genetic Diversity and Structure of Tomato Landraces from the Campania Region (Southern Italy) Uncovers a Distinct Population Identity. Agronomy 2021, 11, 564. [CrossRef]

8. Ganugi, P.; Palchetti, E.; Gori, M.; Calamai, A.; Burridge, A.; Biricolti, S.; Benedettelli, S.; Masoni, A. Molecular Diversity within a Mediterranean and European Panel of Tetraploid Wheat (T. turgidum subsp.) Landraces and Modern Germplasm Inferred Using a High-Density SNP Array. Agronomy 2021, 11, 414. [CrossRef] 
9. López, M.; Gori, M.; Bini, L.; Ordoñez, E.; Durán, E.; Gutierrez, O.; Masoni, A.; Giordani, E.; Biricolti, S.; Palchetti, E. Genetic Purity of Cacao Criollo from Honduras Is Revealed by SSR Molecular Markers. Agronomy 2021, 11, 225. [CrossRef]

10. Birlanga, V.; Acosta-Motos, J.R.; Pérez-Pérez, J.M. Genotype-Dependent Tipburn Severity during Lettuce Hydroponic Culture Is Associated with Altered Nutrient Leaf Content. Agronomy 2021, 11, 616. [CrossRef]

11. Chan-in, P.; Jamjod, S.; Yimyam, N.; Rerkasem, B.; Pusadee, T. Grain Quality and Allelic Variation of the Badh2 Gene in Thai Fragrant Rice Landraces. Agronomy 2020, 10, 779. [CrossRef] 